\title{
Perfil epidemiológico e clínico de pacientes encaminhados para Reabilitação Cardiopulmonar e Metabólica fase II
}

Epidemiological and clinical profile of patients referred for Cardiopulmonary and Metabolic Rehabilitation phase II in Southern Brazil

FisiSenectus . Unochapecó Ano 6, n. 2 - Jul/Dez. 2018 p. 26-37

Patrícia da Silva Klahr. patricia.klahr@gmail.com.

Fisioterapeuta. Mestre e Doutoranda em Ciências da Reabilitação na Universidade Federal de Ciências da Saúde de Porto Alegre (UFCSPA). Diretora Regional Norte-Nordeste de Ciências da Saúde da Laureate Brasil.

Kássia Fabiélen Soares Rocha Oliveira. fabielen.oliveira@hotmail.com.

Fisioterapeuta pelo Centro Universitário FADERGS.

Christian Corrêa Coronel. coronel.christian@hotmail.com.

Fisioterapeuta. Mestre em Ciências da Saúde pelo Instituto de Cardiologia do Rio Grande do Sul e Doutorando em Ciências da Saúde na Universidade Federal de Ciências da Saúde de Porto Alegre (UFCSPA). Diretor do Serviço de Fisioterapia e Centro de Reabilitação Cardiopulmonar e Metabólica, Coordenador e Preceptor da Residência Multiprofissional em Saúde do Instituto de Cardiologia Fundação Universitária de Cardiologia do Rio Grande do Sul e Coordenador e Professor do Curso de Fisioterapia do Centro Universitário La Salle (Unilasalle) em Canoas.

\section{Resumo}

Introdução: As doenças cardiovasculares são consideradas a principal causa de morte no mundo, ocasionando elevadas taxas de morbidade e altos custos governamentais com saúde. Os programas de reabilitação cardíaca introduzidos no Brasil, na década de 1960, centrados na prática de exercício físico, são considerados um processo de restauração das funções físicas e psicossociais em indivíduos com doença cardiovascular, sendo seus benefícios já bem documentados. No entanto, a participação dessas populações ainda é modesta e o perfil epidemiológico dos participantes nos programas de reabilitação cardiopulmonar ainda precisa ser melhor estudado. Objetivo: Descrever o perfil epidemiológico e clínico dos pacientes cardiopatas em Reabilitação Cardiopulmonar e Metabólica fase II. Métodos: Estudo observacional transversal qualitativo, com amostragem por conveniência. 0 teste de normalidade utilizado foi Kolmogorov-Smirnov e a estatística descritiva foi realizada utilizando média e desvio padrão e mediana e intervalos interquartis. Resultados: Participaram 264 pacientes cardiopatas, com idades entre 16 e 89 anos, a maioria da amostra era do sexo masculino (60,6\%) e estavam acima da faixa de peso considerada normal (80,3\%). A etiologia isquêmica (58\%) foi a mais prevalente e os principais fatores de risco foram hipertensão arterial sistêmica, sedentarismo e história familiar. 0 procedimento cirúrgico mais prevalente foi a cirurgia de revascularização do miocárdio 56 (21,2\%) e o tempo mediano entre o procedimento cirúrgico e o início da fase II foi de um mês. Conclusão: 0 perfil epidemiológico e clínico dos pacientes cardiopatas encaminhados para reabilitação fase II a um centro de referência do estado do Rio Grande do Sul é condizente com o perfil de cardiopatas descrito pela literatura quanto à idade, ao gênero, a dados antropométricos e dados clínicos.

\section{Palavras-chave}

Doenças Cardiovasculares; Reabilitação; Exercício.

\section{Fisi乡̌enectus}




\begin{abstract}
Introduction: Cardiovascular diseases are considered the major cause of death in the world, with high morbidity and mortality rates and high government health costs. Cardiac rehabilitation programs introduced in Brazil in the 1960s focused on the practice of physical exercise, are considered a process of restoration of physical and psychosocial functions in individuals with cardiovascular disease, and its benefits have already been documented, but the participation of these populations is still is modest and the epidemiological and clinical profile of participants in cardiopulmonary rehabilitation programs still needs to be better studied. Objective: Describe the epidemiological and clinical profile of patients with cardiovascular diseases that is in Cardiopulmonary and Metabolic Rehabilitation (CPMR) phase II. Methods: This is a qualitative crosssectional observational study, with convenience sampling. The normality test used was Kolmogorov-Smirnov and descriptive statistics were performed using mean and standard deviation and median and interquartile ranges. Results: A 264 patients with heart disease, aged between 16 and 89 years, the majority of the sample were male $(60,6 \%)$ and were above the normal weight range $(80,3 \%)$. The ischemic etiology $(58 \%)$ was the most prevalent and the main risk factors were systemic arterial hypertension, sedentary lifestyle and family history. The most prevalent surgical procedure was myocardial revascularization surgery $56(21,2 \%)$ and the median time between the surgical procedure and the onset of CPMR II was one month. Conclusion: The profile of the patients referred for phase II rehabilitation to a reference center in the state of Rio Grande do Sul is consistent with the epidemiological and clinical profile of cardiopaths described in the literature.
\end{abstract}

\title{
Keyworks
}

Cardiovascular Diseases; Rehabilitation; Exercise.

\section{Introdução}

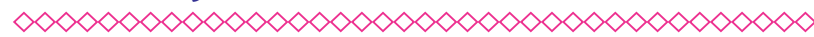

As doenças cardiovasculares são consideradas a principal causa de morte no mundo, possuindo elevadas taxas de morbidade e altos custos governamentais com saúde ${ }^{1,2}$. Segundo a Organização Mundial de Saúde (OMS) as doenças cardiovasculares foram o principal motivo de morte no Brasil em 2011, com uma estimativa de mais de 335 mil óbitos, sendo a região Sul a terceira mais prevalente $^{3}$. No Rio Grande do Sul (RS) as doenças cardiovasculares foram responsáveis, em 2014, por aproximadamente 23 mil mortes ${ }^{4}$.

O encaminhamento dessa população para realizar o exercício físico supervisionado reduz o risco subsequente de morbidade e mortalidade cardiovascular após infarto do miocárdio, intervenção coronária percutânea e cirurgia de revascularização do miocárdio ${ }^{5,6}$, o que contribui para melhorar a qualidade de vida e sobrevida dos pacientes reabilitados.

Assim, os programas de reabilitação cardíaca introduzidos no Brasil na década de 1960, tendo como componente central a prática de exercício físico, são considerados um processo de restauração das funções físicas e psicossociais em indivíduos com doença cardiovascular, tendo seus benefícios já documentados ${ }^{7}$ pela literatura.

No entanto, os programas de Reabilitação Cardiopulmonar e Metabólica (RCPM) são considerados intervenções complexas envolvendo uma equipe multidisciplinar, com intervenções educacionais e comportamentais, e os exercícios físicos contribuem para a maior parte dos resultados ${ }^{2}$.

A fase II da RCPM é a primeira etapa extra-hospitalar, inicia-se imediatamente após a alta e/ ou alguns dias após um evento cardiovascular ou uma descompensação clínica; a duração prevista para esta fase é de três a seis meses; em algumas situações, pode estender-se por mais tempo, com três a cinco sessões semanais ${ }^{\mathbf{8} 9}$. A reabilitação nesta fase tem como principal objetivo melhorar a capacidade funcional do paciente e contribuir para um retorno breve do paciente às suas atividades sociais e laborais, nas melhores condições físicas e emocionais possíveis. ${ }^{\text {8-9-10 }}$

De acordo com a Diretriz de RCPM, o tratamento por meio da reabilitação cardíaca para diversas 
doenças como a coronariopatia, insuficiência cardíaca e hipertensão arterial sistêmica possui evidência nível 1 e recomendação grau $A^{8}$. Porém, a participação dessas populações ainda é modesta nos programas de RCPM, frente aos benefícios já identificados na literatura ${ }^{8}$.

Conhecer o perfil epidemiológico e clínico dos pacientes pode auxiliar a propor novos mecanismos que potencializem a participação das pessoas na RCPM. Isso é do interesse de todos os profissionais de saúde, governos e principalmente do próprio paciente, pois a RCPM pode melhorar a qualidade de vida e ampliar a sobrevida do paciente, assim como reduzir os custos de intervenções hospitalares e com programas para novos e recorrentes eventos cardíacos.

Segundo dados de outros países, como Estados Unidos e Reino Unido, somente 3\% a 20\% com critérios para a realização da reabilitação têm sido encaminhados a esses programas ${ }^{11,12}$, e deste total, a porcentagem de pacientes que realmente aderem à reabilitação cardíaca é de apenas $20 \%$ a $30 \%^{12}$. No Brasil não foram encontrados dados objetivos, portanto, existem lacunas na literatura em relação ao perfil epidemiológico e clínico dos pacientes encaminhados aos programas de RCPM.

É importantíssimo a ampliação do conhecimento no que tange aos dados do perfil epidemiológico e clínico dos pacientes que se encontram inseridos em um programa de RCPM, pois pelo conhecimento destes, há possibilidade de aumentar o encaminhamento, a participação e a adesão dos pacientes na RCPM, o que culmina em redução de custos governamentais, melhora da qualidade de vida dos pacientes, aumento da sobrevida, além da redução de novos eventos. Dessa forma, o presente estudo tem como objetivo descrever o perfil epidemiológico e clínico dos pacientes com doenças cardíacas encaminhados para reabilitação fase II ao Centro de RCPM do Instituto de Cardiologia de Porto Alegre, no Rio Grande do Sul.

\section{Materiais e métodos}

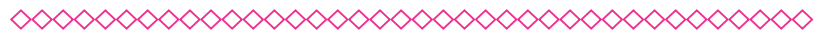

Trata-se de um estudo observacional transversal, predominantemente qualitativo, com coletas de dados quantitativos apenas para compor o perfil clínico. A população amostral foi constituída por todos os sujeitos que ingressaram no Centro de RCPM do Instituto de Cardiologia do Rio Grande do Sul no período de abril de 2012 a abril de 2013, no primeiro ano do serviço. Como critério de inclusão, o participante deveria ter um encaminhamento médico por escrito para a realizar a reabilitação fase II. Não houve critérios de exclusão. 0 projeto foi previamente aprovado pelo Comitê de Ética e Pesquisa do Instituto de Cardiologia do Rio Grande do Sul/Fundação Universitária de Cardiologia sob o número UP. 4846/13.

Ao ingressarem no programa de reabilitação fase II os pacientes foram submetidos a uma anamnese, cuja função foi registrar os principais pontos sobre a sua história pregressa e atual, bem como o uso de medicações. Todas as informações contidas neste estudo foram coletadas na ficha de anamnese/avaliação preenchida com o paciente no seu primeiro dia no centro de RCPM.

Os desfechos primários avaliados foram doença cardíaca, identificada pelo diagnóstico médico e o tempo decorrido desde o evento até o início da reabilitação. Os desfechos secundários avaliados foram sinais vitais, saturação, etnia, dados antropométricos, queixa principal, medicamentos, residência, se ainda exerce função, cidade em que reside e os principais sintomas apresentados.

A avaliação física e funcional dos pacientes está representada em um fluxograma (Figura 1) e incluí a capacidade funcional mensurada com 0 teste de senta e levanta, teste de caminhada de 6 minutos $(\mathrm{TC} 6 \mathrm{~min})^{13} \mathrm{com}$ procedimento padronizado pela ATS, 2000; força de músculos ventilatórios através da pressão inspiratória máxima (Pimáx) e da pressão expiratória máxima (Pemáx) (obtidas na manovacuometria pelo manovacuometro digi-

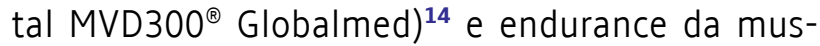
culatura ventilatória pela ventilação voluntária máxima (VVM) mensurada com o ventilômetro de Wright $^{14}\left(\mathrm{OHMEDA}^{\circledR}\right)$, respectivamente.

Também foram coletadas as etiologias, os fatores de risco apresentados pelos pacientes participantes do estudo e os procedimentos cirúrgi$\cos$ aos quais eles foram submetidos. Ainda, com a coleta destes dados, foi possível quantificar 0 V02 indireto dos pacientes. As avaliações foram 
realizadas por um fisioterapeuta treinado e por meio um formulário padronizado para as coletas dos desfechos primários e secundários.

\section{Análise estatística}

$\infty \infty \infty \times \infty \times \infty \times \infty \times \infty \times \infty \times \infty \times \infty \times \infty \times \infty \times \infty \infty \infty$

Realizou-se a análise estatística descritiva pelo programa Statistical Package for the Social Sciences (SPSS), versão 21. Para a análise de normalidade dos dados quantitativos, utilizou-se 0 teste de Kolmogorov-Smirnov. A apresentação dos resultados foi realizada por frequência absoluta e relativa. Já os dados contínuos foram descritos utilizando média e desvio padrão para variáveis com distribuição Gaussiana e mediana e intervalos interquartis para os dados assimétricos.

\section{Resultados}

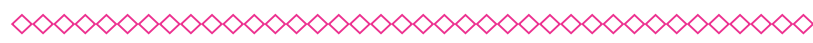

No período de abril de 2012 a abril de 2013, foram atendidos 264 pacientes cardiopatas no centro de RCPM destes, $100 \%$ dos atendimentos foram subsidiados pelo Sistema Único de Saúde (SUS). As idades variaram entre 16 e 89 anos. A proporção de pacientes do sexo masculino $(60,6 \%)$ foi maior do que feminino. Os dados de caracterização da amostra estão disponíveis na Tabela $\mathbf{1}$.

A caracterização clínica e funcional dos pacientes foi apresentada na Tabela 2 . Os dados revelam que em média, a condição dos pacientes encaminhados para RCPM não era grave, muitos estavam estáveis, pois os dados da avaliação de pressão arterial, frequência cardíaca, saturação e até mesmo os percentuais preditos da ventilação voluntária máxima, teste de caminhada de 6 minutos e força muscular ventilatória demonstram isso.

As principais queixas relatadas pelos pacientes, os sintomas e as medicações em uso foram apresentados na Tabela 3. Apenas 20 pacientes $(7,6 \%)$ dos pacientes não estavam medicados para doenças cardíacas durante o processo de reabilitação e a principal queixa referida foi o cansaço.
A etiologia isquêmica foi a mais prevalente, com 153 pacientes (58\%), seguida de miocardiopatia, com 24 pacientes (9\%), e valvular com 11 pacientes $(4,2 \%)$, entre todos os diagnósticos.

Os principais fatores de risco identificados na amostra foram à hipertensão arterial sistêmica (HAS), o sedentarismo e a história familiar e seus respectivos valores, e estão apresentados na Tabela 4 .

A maioria, 152 pacientes (57,5\%), foi encaminhada para a RCPM para controle de fatores de risco sem submissão a procedimento cirúrgico prévio. Naqueles submetidos a procedimento prévio, a cirurgia de revascularização do miocárdio foi a mais prevalente, com 56 pacientes $(21,2 \%)$. 0 tempo mediano entre o procedimento cirúrgico e o início da RCPM fase II foi de um mês. Os demais dados estão apresentados na Tabela 5.

\section{Discussão}

$\infty \times \infty \times \infty \times \infty \times \infty \times \infty \times \infty \times \infty \times \infty \times \infty \times \infty \times \infty \infty \infty$

Assim como em outros estudos ${ }^{15-17}$, a média de idade dos pacientes encaminhados a RCPM é de aproximadamente 60 anos, demonstrando que as doenças cardiovasculares podem acometer os indivíduos ao longo de toda vida, porém, geralmente, são doenças progressivas que avançam com a idade, e muitas vezes a progressão é lenta, estando associada sua maior debilidade com a terceira idade $^{18,19,20}$. A maioria dos pacientes eram do sexo masculino e apresentavam excesso de peso corporal, o que também está descrito na literatura para pacientes com doenças cardíacas em reabilitação ${ }^{15,17}$. Diferentemente dos estudos americanos, a proporção de pacientes da etnia negra foi menor, uma vez que a etnia predominante dessa região do estado é branca devido à sua colonização por imigrantes italianos, alemães e portugueses ${ }^{16}$. Entre os participantes, 69,3\% residem em Porto Alegre e os demais, nas cidades próximas.

A maioria dos pacientes apresentaram valores normais de pressão arterial, frequência cardíaca e saturação parcial de oxigênio, uma vez que, dos 264 pacientes incluídos no estudo, 244 estavam medicados para suas respectivas doenças e os fármacos mantiveram estáveis os sinais vitais. Dentre 
as classes medicamentosas mais utilizadas, assim como no estudo de Muela ${ }^{15}$, encontram-se com maior prevalência os betabloqueadores $(89,8 \%)$, os antiplaquetários $(80,7 \%)$ e as estatinas $(71,6 \%)$.

A etiologia cardíaca mais frequente diagnosticada pelos médicos entre os pacientes participantes da pesquisa foi a de origem isquêmica, a qual também é a mais prevalente descrita em outros estudos $^{15}$, o que corrobora com a maior prevalência de cirurgias de revascularização do miocárdio. Os três principais fatores de risco relatados no nosso estudo foram a HAS, o sedentarismo e a história familiar positiva para problemas cardíacos. No estudo de Muela ${ }^{15}$, as maiores prevalências de fatores de risco foram, de maneira decrescente: HAS, dislipidemias e história familiar de doença arterial coronariana (DAC) positiva.

0 sedentarismo descrito em nosso estudo como um dos fatores de risco predominante corrobora com as duas principais queixas referidas: o cansaço e a dispneia. Também a má qualidade do sono está associada ao cansaço. Araujo et al. ${ }^{21}$ descreve, em seu estudo, a prevalência de $8 \%$ a $10 \%$ na população em geral, e de $50 \%$ a $70 \%$ na população de idosos, que a má qualidade do sono está fortemente associada às doenças cardiovasculares, assim, pacientes que possuem uma meIhor qualidade do sono têm melhores resultados na RCPM ${ }^{21}$ por apresentarem menor cansaço.

Os participantes deste estudo no momento da avaliação apresentaram os valores preditos da VVM, teste de sentar e levantar (SL), Pimáx, Pemáx, TC6min abaixo do esperado para seu biotipo, a grande maioria estava com sua capacidade funcional, força muscular e endurance ventilatório reduzidos. Em nosso estudo, observamos o tempo mediano de encaminhamento para a RCPM após procedimento cirúrgico de um mês. As diretrizes de reabilitação cardíaca, ${ }^{8,22}$ sugerem que o tempo ideal entre a alta hospitalar e o início da RCPM fase II seja entre uma e quatro semanas, dependendo do tipo de evento. Semelhante ao nosso achado, um estudo americano ${ }^{16}$ observou que o tempo mediano de encaminhamento foi de 35 dias. Os autores constataram, ainda, uma diminuição na taxa de mortalidade dos pacientes encaminhados porém, concluíram que era subutilizado o serviço, podendo ser ampliado o número de pacientes encaminhados e preferencialmente potencializada a RCPM com um encaminhamento precoce em até 10 dias após a alta hospitalar, que corroboraria na adesão e aderência ao tratamento ${ }^{16}$.

Alguns estudos sugerem que a cada dia de atraso no início da RCPM fase II, há uma diminuição de $1 \%$ na aderência e participação no programa ${ }^{16}$. A RCPM é o somatório das atividades necessárias para garantir aos pacientes portadores de cardiopatia a melhor condição física, mental e social. Os pacientes que aderem a programas de RCPM apresentam inúmeras mudanças hemodinâmicas, metabólicas, vasculares e psicológicas que estão associadas a um maior controle dos fatores de risco e a melhora da qualidade de vida $8,15,23,24$.

Segundo dados americanos apenas $14 \%$ a $35 \%$ dos pacientes que poderiam ser encaminhados a RCPM são de fato encaminhados para os serviços ${ }^{11,12,15,25,26}$ e somente $31 \%$ dos pacientes submetidos à cirurgia de revascularização do miocárdio (CRM) ${ }^{16,27}$. Atualmente, existem apenas 160 serviços que realizam RCPM na América do Sul, estes distribuídos em nove dos dez países que compõe a Sociedade Sul Americana de Cardiologia, existindo apenas um serviço para cada 2.319.312 habitantes ${ }^{28}$. Os estudos sugerem que esse baixo número deve-se à escassez de serviços estruturados, poucos encaminhamentos por parte dos médicos assistentes devido ao desconhecimento dos reais benefícios da RCPM e, principalmente, à ausência de cobertura deste serviço pela maioria dos convênios ${ }^{15,16,24,28}$.

Ao longo dos anos, a RCPM vem demonstrando modificações benéficas nos fatores de risco e reduções na morbimortalidade cardiovascular em um cenário considerado excelente em termos de custo-efetividade terapêutica ${ }^{15,29}$. Os programas de RCPM, além de promover uma redução no índice de reinternação hospitalar por descompensação (três vezes menor), são capazes de aumentar a expectativa de vida em cerca de dois anos ${ }^{15}$. No entanto, mesmo demonstrando resultados positivos na recuperação dos pacientes e redução de gastos em serviços de saúde, o número de centros de reabilitação cardíaca ainda é escasso, principalmente na rede pública ${ }^{15,24,28}$, e os existentes ainda são subutilizados ${ }^{15,16,24,28}$. 


\section{Limitações}

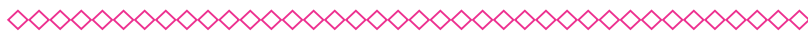

A principal limitação do nosso estudo foi a ausência do teste de esforço cardiopulmonar para mensurar os gases expirados, o limiar anaeróbico e o V02 máximo destes pacientes. Além disso, a ausência de uma avaliação da qualidade do sono (polissonografia) também foi um limitador significativo para estabelecer a associação válida entre o cansaço e a má qualidade do sono.

\section{Conclusão}

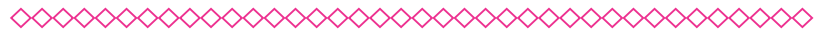

O perfil epidemiológico e clínico dos 264 pacientes com doenças cardíacas encaminhados para reabilitação fase II ao Centro de RCPM do Instituto de Cardiologia de Porto Alegre, no Rio Grande do Sul, é condizente com o perfil epidemiológico e clínico de pacientes descrito nas demais regiões brasileiras. A maioria dos participantes da amostra era do sexo masculino $(60,6 \%)$ e estavam acima da faixa de peso considerada normal (80,3\%). A etiologia isquêmica (58\%) foi a mais prevalente. Os principais fatores de risco foram: hipertensão arterial sistêmica, sedentarismo e história familiar. 0 procedimento cirúrgico mais prevalente foi a cirurgia de revascularização do miocárdio, com 56 pacientes ( $21,2 \%$ ), e o tempo mediano entre o procedimento cirúrgico e o início da fase II foi de um mês. 0 objetivo deste estudo limitou-se a descrever o perfil epidemiológico e clínico dos pacientes portanto, sugere-se que novos estudos verifiquem as associações entre os desfechos clínicos e funcionais e a realização de estudos de coorte que possam acompanhar o comportamento destes desfechos ao longo da reabilitação cardiopulmonar e metabólica.

\section{Referências}

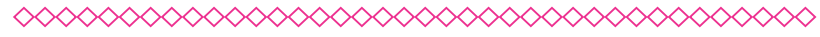

1. Cardiac Care Network. The Ontario cardiac rehabilitation pilot project. Report and recommendations. 2002.
2. Souza CA, Santos RZ, Lineburger AA, Benetti M. RCPM na atenção primária em saúde: é possível? R. bras. Ci. e Mov. 2015;23(1):164-71.

3. World Health Organization. Cardiovascular diseases. 2010. http://www.who.int/mediacentre/ factsheets/fs317/en/index.html.

4. Secretaria da Saúde. Plano Estadual de Saúde 2016-2019 do Rio Grande do Sul. http://www. saude.rs.gov.br/upload/1471009389_PES\%20 2016-2019\%20-\%2012\%2008.pdf. Acesso em 20 jan. 2017.

5. Heran BS, Chen JM, Ebrahim S, Moxham $T$, Oldridge $N$, Rees K, et al. Exercise-based cardiac rehabilitation for coronary heart disease. Cochrane Database Syst Rev. 2011; 6(7):CD001800

6. Goel K, Lennon RJ, Tilbury RT, Squires RW, Thomas RJ. Impact of cardiac rehabilitation on mortality and cardiovascular events after percutaneous coronary intervention in the community. Circulation. 2011;123(21):2344-52.

7. Thomas RJ, King M, Lui K, Oldridge N, Piña IL, Spertus J, et al. AACVPR/ACC/AHA 2007 performance measures on cardiac rehabilitation for referral to and delivery of cardiac rehabilitation/secondary prevention services endorsed by the American College of Chest Physicians, American College of Sports Medicine, Preventive Cardiovascular Nurses Association, and the Society of Thoracic Surgeons. J Am Coll Cardiol. 2007;50(14):1400-33.

8. Carvalho $T$, Cortez AA, Ferraz A, Nóbrega ACL, Brunetto AF, Herdy AH, et al. Sociedade Brasileira de Cardiologia. Diretriz de RCPM: aspectos práticos e responsabilidades. Arq Bras Cardiol. 2006;86(1):74-82.

9. Herdy AH, López-Jiménez F, Terzic CP, Milani M, Stein $\mathrm{R}$, et al. Diretriz Sul-Americanade Prevenção e Reabilitação Cardiovascular. Sociedade Brasileira de Cardiologia. 2014;103(2, Supl. 1):1-27.

10. Chou CL, Lee SH, Su YT, Hong SY, Pan BR, Chan RC. Impact of Phase II cardiac rehabilitation on abnormal heart rate recovery - Journal of the Chinese Medical Association. 2014;77(9):482-6. 
11. Dalal H, Evans PH, Campbell JL. Recent developments in secondary prevention cardiac rehabilitation after acute myocardial infarction. BMJ.2004;328(7441):693-97.

12. Sundararajan V, Bunker SJ, Begg S, Marshall $\mathrm{R}$, McBurney $\mathrm{H}$. Attendance rates and outcomes of cardiac rehabilitation in Victoria, 1998. Med J Aust. 2004;180(6):268-71.

13. American Thoracic Society. Statement guidelines for the six-minute walk test. Am F Respir Crit Care Med 2002;166:111-117.

14. American Thoracic Society/European Respiratory Society. ATS/ERS. Statement on respiratory muscle testing. Am F Respir Crit Care Med 2002;166(4):518-624.

15. Muela HCS, Bassan R, Serra SM.

Avaliação dos Benefícios Funcionais de um Programa de Reabilitação Cardíaca Rev Bras Cardiol.2011;24(4):241-50.

16. Pack QR, Mansour M, Barboza JS, Hibner BA, Mahan MG, Ehrman JK, et al. An early appointment to outpatient cardiac rehabilitation hospital discharge improves attendance at orientation: a randomized, single-blind, controlled trial. Circulation, 2013;127(3):349-55.

17. Rossi RC, Vanderlei FM, Medina LAR, Pastre $C M$, Padovani CR, Vanderlei, LCM. Influência do perfil clínico e sociodemográfico na qualidade de vida de cardiopatas submetidos à reabilitação cardíaca. ConScientiae Saúde, 2011;10(1):59-68.

18. Andrade JP, Bocchi EA, Braga FGM, Ferreira SMA, Rohde LEP, Oliveira WA, et al. III Diretriz brasileira de insuficiência cardíaca crônica. Arq Bras Cardiol. 2009; 93(1): 1-71.

19. Berry JRS, Cunha AB. Avaliação dos efeitos da reabilitação cardíaca em pacientes pós-infarto do miocárdio. Rev Bras Cardiol. 2010;23(2):101-10.

20. Benetti M, Araujo CLP, Santos RZ. Aptidão cardiorrespiratória e qualidade de vida pósinfarto em diferentes intensidades de exercício. Arq Bras Cardiol. 2010;95(3):399-404.

21. Araujo PAB, Sties SW, Wittkopf PG, Netto AS, Gonzáles AI, Lima DP, et al. Índice da qualidade do sono de Pitt Sburgh para uso na RCPM. Revista Brasileira de Medicina do Esporte. 2015; 21

(6):472-5.

22. Carvalho T, Curi ALH, Andrade DF, Singer JM, Benetti M, Mansur AJ. Reabilitação cardiovascular de portadores de cardiopatia isquêmica submetidos a tratamento clínico, angioplastia coronariana transluminal percutânea. Arq Bras Cardiol. 2007;88(1):72-8.

23. Perk J, Backer GD, Gohlke H, Graham I, Reiner $Z$, Verschuren WMM, et al. European guidelines on cardiovascular disease prevention in clinical practice (version 2012). European Heart Journal. 2012;33(1):1635-1701.

24. Cortés 0 , Arthur HM. Determinants of referral to cardiac rehabilitation programs in patients with coronary artery disease: a systematic review. Am Heart J. 2006;151(2):249-56.

25. Ades PA. Cardiac rehabilitation and secondary prevention of coronary heart disease. N Engl J Med. 2001;345(12):892-902.

26. Centers for Disease Control and Prevention (CDC). Receipt of outpatient cardiac rehabilitation among heart attack survivors - United States, 2005. MMWR Morb Mortal Wkly Rep. 2008;57(4):89-94.

27. Suaya JÁ, Shepard DS, Normand SL, Ades PA, Prottas J, Stason WB. Use of cardiac rehabilitation by Medicare beneficiaries after myocardial infarctation or coronary bypass surgery. Circulation, 2007;116(15):1653-62.

28. Cortes-Bergoderi M, Lopez-Jimenez F, Herdy AH, Zeballos C, Anchique C, Santibañez $C$, et al. Availability and characteristics of cardiovascular rehabilitation programs in South America. J. Cardiopulm Rehabil Prev. 2013 JanFeb;33(1):33-41.

29. Moreira PBB. Custo-efetividade de programas de reabilitação cardiovascular. Jornal do Departamento de Ergometria e Reabilitação Cardíaca. 2004;27:14-6. 


\section{Anexos}

Figura 1 - Fluxograma do processo de avaliação dos pacientes encaminhados reabilitação cardiopulmonar e metabólica, fase II.

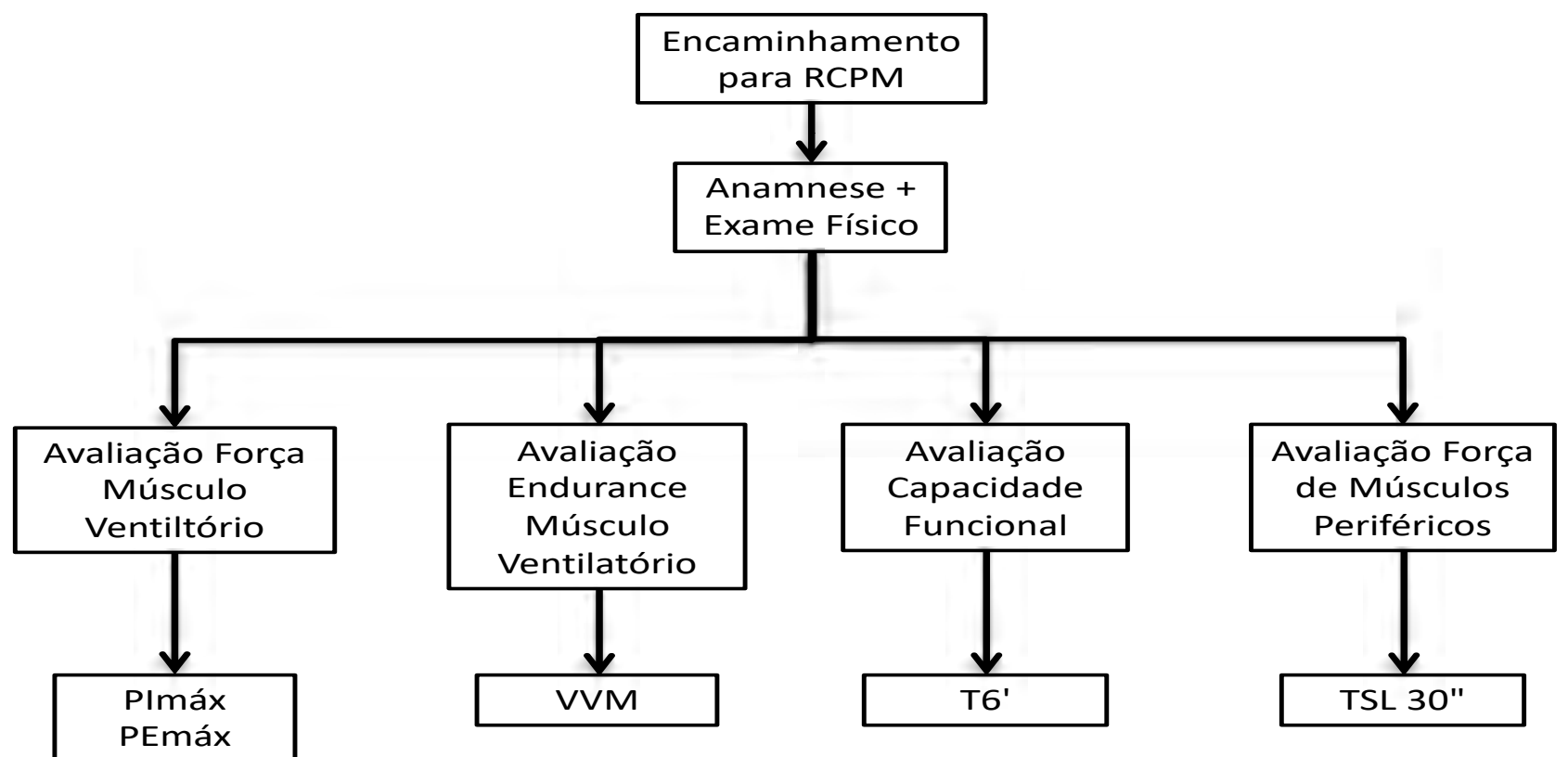

Legenda: RCPM - reabilitação cardiopulmonar e metabólica; PImáx - pressão inspiratória máxima; PEmáx - pressão expiratória máxima; VVM - ventilação voluntária máxima; T6'- teste de caminhada de seis minutos e TSL 30"- teste de senta e levanta de 30 segundos 
Tabela 1 - Caracterização da amostra dos 264 pacientes em Reabilitação Cardiopulmonar e Metabólica, fase II.

\begin{tabular}{llcc} 
Variáveis & & DP \\
Idade (anos) & & 62,23 & $(12,35)$ \\
Peso (Kg) & & 80,89 & $(16,67)$ \\
Altura (m) & & 1,65 & $(0,09)$ \\
Sexo-n(\%) & Masculino & & \\
& Feminino & 160 & $(60,6)$ \\
& & 104 & $(39,4)$ \\
Etnia-n(\%) & Branca & & \\
& Negra & 223 & $(84,5)$ \\
& Outras & 32 & $(12,1)$ \\
& & 9 & $(3,4)$ \\
Indice de Massa Corporal - n (\%) & Excesso de Magreza & & $(1,5)$ \\
& Peso normal & 4 & $(18,2)$ \\
& Excesso de Peso & 48 & $(38,8)$ \\
& Obesidade (Grau I) & 102 & $(26,9)$ \\
& Obesidade (Grau II) & 71 & $(10,2)$ \\
& Obesidade (Grau III) & 27 & $(4,5)$ \\
& & 12 & $(33)$ \\
Aidade que reside-n(\%) & & 87 & $(39,8)$ \\
& & 105 & $(69,3)$ \\
& & & 183 \\
\hline
\end{tabular}

Legenda: $\boldsymbol{X}$ - média; DP - refere-se ao desvio-padrão, n=frequência absoluta e \%=frequência relativa. 
Tabela 2 - Caracterização clínica e funcional da amostra dos 264 pacientes.

\begin{tabular}{lcc} 
Variáveis & X & DP \\
Pressão arterial sistólica (mmHg) & 125,32 & $(19,35)$ \\
Pressão arterial diastólica $(\mathrm{mmHg})$ & 73,58 & $(12,11)$ \\
Frequência cardíaca (bpm) & 72,48 & $(13,18)$ \\
Saturação parcial de O $(\%)$ & 96,30 & $(10,54)$ \\
Vo2 Indireto & 14,99 & $(5,61)$ \\
Ventilação Voluntária Máxima (L) & 50,89 & $(23,48)$ \\
Percentual predito da ventilação voluntária máxima & 89,29 & $(46,81)$ \\
Senta e levanta & 11,33 & $(3,51)$ \\
Pressão Inspiratória Máxima & 74,23 & $(33,08)$ \\
Percentual predito da pressão inspiratória máxima & 78,81 & $\pm 37,95$ \\
Pressão Expiratória Máxima & 87,98 & $\pm 42,91$ \\
Percentual predito da pressão expiratória máxima & 90,61 & $\pm 49,29$ \\
Teste de caminhada de 6 minutos (m) & 406,29 & 108,18 \\
Percentual predito do teste de caminhada de 6 minutos & 81,87 & $\pm 35,69$ \\
Tempo do evento até a reabilitação - (meses) (mediana - Q1-Q3) & 1 & $(1-3)$ \\
\hline
\end{tabular}

Legenda: $\boldsymbol{X}$ - média; DP - refere-se ao desvio-padrão, n=frequência absoluta e \%=frequência relativa. 
Tabela 3 - Caracterização das queixas principais,

de sintomas e medicamentos da amostra dos 264 pacientes.

\begin{tabular}{|c|c|c|c|}
\hline Variáveis & & $\mathbf{n}$ & $\%$ \\
\hline \multicolumn{4}{|l|}{ Queixa principal -n(\%) } \\
\hline & Cansaço & 79 & $(29,9)$ \\
\hline & Dispneia & 26 & $(9,8)$ \\
\hline & Cansaço associado à dispneia & 29 & $(11)$ \\
\hline & Dor em membros inferiores & 13 & $(4,9)$ \\
\hline & Dor torácica & 13 & $(4,9)$ \\
\hline \multicolumn{4}{|l|}{ Principais sintomas - $n(\%)$} \\
\hline Assintomático & & 72 & $(27,3)$ \\
\hline \multirow[t]{5}{*}{ Dispneia } & & 115 & $(43,6)$ \\
\hline & NYHA CFI & 38 & $(14,4)$ \\
\hline & NYHA CFII & 42 & $(15,9)$ \\
\hline & NYHA CFIII & 31 & $(11,7)$ \\
\hline & NYHA CFIV & 4 & $(1,5)$ \\
\hline Tosse & & 64 & $(24,2)$ \\
\hline Ortopnéia & & 7 & $(2,7)$ \\
\hline Dispneia Paroxística Noturna & & 23 & $(8,7)$ \\
\hline Dor torácica & & 56 & $(21,2)$ \\
\hline \multirow[t]{5}{*}{ Dor torácica típica } & & 30 & $(11,4)$ \\
\hline & Classe Funcional I & 7 & $(2,7)$ \\
\hline & Classe Funcional II & 12 & $(4,5)$ \\
\hline & Classe Funcional III & 7 & $(2,7)$ \\
\hline & Classe Funcional IV & 4 & $(1,5)$ \\
\hline Palpitações & & 75 & $(28,4)$ \\
\hline Tontura & & 97 & $(36,7)$ \\
\hline Síncope & & 13 & $(4,9)$ \\
\hline Claudicação Intermitente & & 26 & $(9,8)$ \\
\hline $\begin{array}{l}\text { Medicados para a doença } \\
\text { cardíaca - } n(\%)\end{array}$ & & 244 & $(92,4)$ \\
\hline \multicolumn{4}{|l|}{ Principais Drogas - n(\%) } \\
\hline & Diuréticos & 92 & $(34,8)$ \\
\hline & $\begin{array}{l}\text { Inibidores da enzima conversora } \\
\text { da angiotensina }\end{array}$ & 97 & $(36,7)$ \\
\hline & $\begin{array}{l}\text { Antagonista Receptores } \\
\text { Angiotensina }\end{array}$ & 53 & $(20,1)$ \\
\hline & Betabloqueadores & 162 & $(61,4)$ \\
\hline & Anticoagulantes & 93 & $(35,2)$ \\
\hline & Antiarritmicos & 130 & $(49,2)$ \\
\hline & Estatinas & 144 & $(54,5)$ \\
\hline & Biguanida & 36 & $(13,6)$ \\
\hline & Nitrato & 38 & $(14,4)$ \\
\hline & Outras & 75 & $(28,4)$ \\
\hline
\end{tabular}

Legenda: DP - refere-se ao desvio-padrão, n=frequência absoluta e \%=frequência relativa. 
Tabela 4 - Principais fatores de risco apresentados pelos 264 pacientes.

\begin{tabular}{lcc} 
Fator de risco & \multicolumn{2}{c}{ Sim } \\
Dislipidemia & 150 & 56,8 \\
Hipertensão arterial sistêmica & 210 & 79,5 \\
Sedentarismo & 186 & 70,5 \\
Diabete Mellitus & 78 & 29,5 \\
Obesidade & 73 & 27,7 \\
Tabagismo ativo & 17 & 6,4 \\
Ex-tabagistas & 116 & 44,0 \\
Etilismo ativo & 34 & 12,9 \\
Ex-etilista & 16 & 6,1 \\
História familiar positiva & 173 & 65,5 \\
\hline
\end{tabular}

Legenda: Valores expressos em frequência absoluta (n) e relativa(\%).

(clique para voltar ao texto)

Tabela 5 - Principais procedimentos cirúrgicos realizados previamente a RCPM.

\begin{tabular}{lcc} 
Procedimento Cirúrgico & \multicolumn{2}{c}{ Sim } \\
Não realizaram procedimento cirúrgico & 152 & 57,5 \\
Reperfusão Hemodinâmico (ACTP e/ou Stent) & 24 & 9,1 \\
Cirurgia de Revascularização do Miocárdio & 56 & 21,2 \\
Correção Cardiopatia Congênita & 1 & 0,4 \\
Aortoplastia & 4 & 1,5 \\
Valvuloplastia & 8 & 3,0 \\
Cateterismo & 14 & 5,4 \\
Marcapasso & 4 & 1,5 \\
Transplante cardíaco & 1 & 0,4 \\
\hline Total & 264 & 100,0 \\
\hline
\end{tabular}

Legenda: Valores expressos em frequência absoluta (n) e relativa (\%). RCPM - Reabilitação Cardiopulmonar e Metabólica. 